\title{
Electores, partidos y la caja de Pandora
}

\author{
Alvaro Artiga-González
}

\begin{abstract}
Resumen
En este artículo se analiza, por un lado, la respuesta de la ciudadanía -en términos de participación electoral- a los llamados de los partidos políticos y del Tribunal Supremo Electoral en las recientes elecciones del 12 de marzo. Por otro lado, la traducción de esa respuesta en asignación de puestos de elección pública. El abordaje analítico es a nivel agregado y no a nivel de individuos. Interesa describir la distribución territorial de la participación electoral y de las preferencias partidistas y cómo opera sobre ellas el sistema electoral a la hora del reparto de los escaños.
\end{abstract}

\section{Electores}

Atendiendo a la participación, las elecciones del 12 de marzo serán recordadas porque a pesar del esfuerzo, real o aparente, del Tribunal Supremo Electoral (TSE) y de algunos partidos políticos, más los llamados desde diversos programas de radio y televisión, el nivel de participación registrado fue el más bajo desde 1982, el cual alcanzó una cifra entre el 35 y 40 por ciento de la población en el padrón electoral ${ }^{2}$. Es cierto que se trataba de unas elecciones de menor importancia en comparación con las elecciones presidenciales. Razonando de esta forma se podría haber justifi- cado de antemano que la concurrencia electoral sería menor a la registrada en 1999. Pero lo que ha llamado la atención no es el descenso esperado en la participación, sino el nivel de ésta. Si bien no se cuestiona la legitimidad de los elegidos, sí queda en entredicho su representatividad.

Podría intentar ofrecer algunas explicaciones e interpretaciones de este fenómeno. Sin embargo, el Instituto Universitario de Opinión Pública de la UCA (IUDOP) y sus encuestas han venido postulando desde hace varios años que en la base del ausentismo $y$, por tanto, de la tendencia hacia una menor participación electoral, está el desinterés, la

1. Profesor e investigador del Departamento de Sociología y Ciencias Políticas de la UCA. E-mail: alvart@buho.uca.edu.sv

2. En la medida en que no toda la población registrada en el padrón electoral es la población con derecho a voto (los mayores de 18 años), otros cálculos de la participación oscilan entre el 30 y el 35 por ciento (Proceso 894). 
apatía, la desconfianza y la falta de credibilidad en la política y los políticos por parte de una creciente proporción de la ciudadanía salvadoreña. Me sumo a estas razones del IUDOP. Además, en un análisis comparativo del comportamiento electoral de los centroamericanos entre 1980 y 1997, señalé que también ha ocurrido un desgaste del procedimiento electoral en países como Guatemala y El Salvador (países donde más elecciones hubo en dicho período), como consecuencia de promesas incumplidas de manera reiterada, de ineficacia percibida para lograr por ese medio los fines prometidos, concretamente para lograr una pacificación que sólo llegó luego de prolongados procesos de diálogo y negociación ... las elecciones del 12 de marzo serán recordadas porque a pesar del esfuerzo, real o aparente, del Tribunal Supremo Electoral (TSE) y de algunos partidos políticos, más los llamados desde diversos programas de radio y televisión, el nivel de participación registrado fue el más bajo desde 1982, el cual alcanzó una cifra entre el 35 y 40 por ciento de la población en el padrón electoral. ¿los candidatos o los partidos? Si son los candidatos los que constituyen dicho criterio se esperaría que se registrara mucho voto cruzado, es decir, que los electores votaran a un partido en la elección nacional y a otro en la elección local. $\mathrm{Si}$, por el contrario, lo que orienta al elector es el partido, entonces se esperaría poco voto cruzado, es decir, independientemente del tipo de elección, el voto sería para el mismo partido. Sin embargo, un análisis de este tipo consumiría tantas páginas que no habría espacio en este artículo para observar a los partidos y destapar la caja de Pandora. Dejaré para otra ocasión ese análisis. Aquí me centraré más en una descripción de la participación desde el punto de entre las partes en conflicto (Artiga-González, 1997a). Considero que esta ineficacia percibida también ha contribuido en esta ocasión, pues desde 1994 hasta la fecha algunos políticos y partidos han venido ofreciendo que, a cambio de un voto a su favor, la solución de los problemas económicos y de seguridad pública está más cercana; cosa que no parece ser cierta para los electores que han dejado de votar y para aquellos que, habiendo alcanzado la edad de votar, se han abstenido de ejercer ese derecho.

La simultaneidad de elecciones de diputados a la Asamblea Legislativa y de concejos municipales es una magnífica oportunidad para estudiar el comportamiento electoral, ya sea de los que deciden votar o no, ya sea de los que deciden por quién volar. Se trata de elecciones simultáneas, pero de distinto nivel. Las primeras son nacionales (se elije a representantes de la nación), en tanto que las segundas son locales (se elije a los representantes en los gobiernos municipales). En estas condiciones se podría indagar sobre la existencia de una o dos lógicas de comportamiento electoral. Es decir, podría resultar interesante dilucidar cuál es el criterio que define la decisión del votante: vista de las circunscripciones electorales.

\subsection{Participación electoral por circunscripcio- nes}

¿Se puede decir que la participación electoral de los salvadoreños se distribuye homogéneamente sobre todo el país? ¿ $O$ existen diferencias entre departamentos? ¿Y qué sucede a nivel de los municipios? ¿Existen diferencias entre éstos en cuanto a la participación y el ausentismo? El Cuadro 1 presenta información a nivel departamental, que nos permite responder las dos primeras preguntas. Por su parte, el Cuadro 2 se refiere a la participación en las catorce cabeceras departamentales.

Como puede observarse en ambos cuadros, la participación electoral en el 2000 no ha sido homogénea. Existen diferencias entre departamentos y cabeceras. En general, la participación a nivel departamental es mayor que la registrada a nivel de sus respectivas cabeceras. Para los primeros, la participación está siempre arriba del 30 por ciento, no así para las cabeceras, donde La Unión está por debajo de este umbral. En ambos casos, el grueso oscila entre el 30 y el 40 por ciento distribuido de 
forma heterogénea. Finalmente, mientras cuatro departamentos superan el $\mathbf{4 0}$ por ciento de participación, únicamente dos cabeceras superan este umbral: Nueva San Salvador y Chalatenango. De éstas, sólo Chalatenango muestra un comportamiento coherente al superar dicho umbral a nivel departamental y de cabecera. En términos globales, la circunscripción departamental con menor participación fue La Unión -con un 34.5 por ciento- y la de mayor participación fue Cha- latenango - con un 47.1 por ciento-. En cuanto a las cabeceras, la de menor participación fue también La Unión (29.3 por ciento) y la más participativa resultó ser Chalatenango (44.5 por ciento). Es llamativo el hecho de que ambas circunscripciones definan los umbrales mínimo y máximo de participación en ambos niveles, lo cual muestra un comportamiento electoral coherente de sus respectivos electorados.

\section{Cuadro 1}

Participación en elecciones legislativas por departamento, 2000

\begin{tabular}{lrrrr}
\hline & & & \multicolumn{2}{c}{ Participación } \\
\cline { 4 - 5 } Departamento & Población & Electorado & Absoluta & $\%$ \\
\hline San Salvador & $1,985,294$ & 965,371 & 370,765 & 38.4 \\
Santa Ana & 551,259 & 306,492 & 105,551 & 34.4 \\
San Miguel & 480,276 & 244,422 & 88,343 & 36.1 \\
La Libertad & 682,092 & 347,751 & 136,814 & 39.3 \\
Usulután & 338,332 & 195,132 & 74,997 & 38.4 \\
Sonsonate & 450,116 & 246,292 & 95,208 & 38.7 \\
La Unión & 289,021 & 135,917 & 46,858 & 34.5 \\
La Paz & 292,887 & 164,446 & 68,843 & 41.9 \\
Chalatenango & 196,583 & 114,068 & 53,672 & 47.1 \\
Cuscatlán & 202,951 & 115,374 & 46,749 & 40.5 \\
Ahuachapán & 319,780 & 161,422 & 60,920 & 37.7 \\
Morazán & 173,499 & 96,822 & 42,495 & 43.9 \\
San Vicente & 161,105 & 87,336 & 34,265 & 39.2 \\
Cabañas & 152,842 & 83,908 & 30,862 & 36.8 \\
\hline Totales & $6,276,037$ & $3,264,753$ & $1,256,342$ & 38.5 \\
\hline
\end{tabular}

Fuentes: Población, DIGESTYC (1996); electorado: El Diario de Hoy, 12 de marzo de 2000; y participación absoluta: Tribunal Supremo Electoral (2000a).

Llama la atención también que la participación no parece guardar relación alguna con el tamaño de la circunscripción medido en términos de la población que la habita. Una hipótesis que se utiliza en la ciencia política para estudiar la participación y la abstención electoral postula que, a medida que crece la circunscripción, la votación tiende a ser menor porque la presión social para ejercer el voto es mayor en los lugares pequeños (Delgado, 1997; Justel, 1995). No parece ser este el caso en El Salvador, pues circunscripciones pequeñas registran niveles semejantes de participación a los registrados en las circunscripciones más grandes. Para profundizar un poco más en el análisis agregado de la participación electoral en El Salvador podríamos hacemos la siguiente pregunta: ¿existe 
alguna relación entre estos niveles de participación y las características socioeconómicas de la población de cada circunscripción? El Cuadro 3 muestra información que nos ayuda a responder esta cuestión'.

\section{Cuadro 2 \\ Participación en elecciones legislativas por cabeceras, 2000}

\begin{tabular}{lrrrr}
\hline & & & \multicolumn{2}{c}{ Participación } \\
\cline { 4 - 5 } Cabecera & Población & Electorado & Absoluta & $\%$ \\
\hline San Salvador & 479,605 & n.d. & 124,245 & n.d. \\
Santa Ana & 248,963 & 149,412 & 47,095 & 31.5 \\
San Miguel & 239,038 & 122,930 & 38,145 & 31.0 \\
Nueva San Salvador & 158,207 & 84,589 & 35,704 & 42.2 \\
Usulután & 69,099 & 49,139 & 15,228 & 31.0 \\
Sonsonate & 96,772 & 62,440 & 20,975 & 33.6 \\
La Unión & 40,371 & 31,565 & 9,262 & 29.3 \\
Zacatecoluca & 62,352 & 44,986 & 16,409 & 36.5 \\
Chalatenango & 30,096 & 18,673 & 8,401 & 44.5 \\
Cojutepeque & 53,122 & 37,159 & 12,117 & 32.6 \\
Ahuachapán & 107,534 & 55,823 & 19,138 & 34.3 \\
San Francisco Gotera & 21,181 & 13,428 & 5,162 & 38.4 \\
San Vicente & 50,751 & 33,006 & 10,852 & 39.9 \\
Sensuntepeque & 41,068 & 24,157 & 7,501 & 31.1 \\
\hline Total** & $1,218,554$ & 727,307 & 245,989 & 33.8 \\
\hline
\end{tabular}

* Votación en elección del Concejo Municipal. Opté por este dato por no contar con la votación de la cabecera para la elección de la Asamblea Legislativa.

** Sin incluir San Salvador.

n.d. = No hay dato.

Fuente: Población, DIGESTYC (1996); electorado: La Prensa Gráfica, 10 de marzo de 2000; y participación absoluta: Tribunal Supremo Electoral (2000b)

En primer lugar, puede notarse que las circunscripciones que están por arriba de la media nacional en participación tienden a estar por debajo de los índices nacionales de educación e ingreso, excepto La Libertad, en ambos, y Cuscatlán, para educación. En segundo lugar, Chalatenango es la circunscripción más participativa, que supera por casi 8 puntos porcentuales a San Salvador y $\mathbf{L a}$ Libertad, que son las circunscripciones más ilustradas y más ricas del país. Por tanto, no parece haber una fuerte relación directa entre educación, ingreso y participación electoral. De hecho, al calcular el índice de correlación de Pearson da valores de 0.0001 para educación-participación y 0.035 para ingreso-participación. Este resultado sugiere que no es en las variables estructurales donde hay que buscar la explicación de la participación electoral agregada. Llama la atención que sean Chalatenango y Morazán las circunscripciones más participativas, pues se sabe que en la his-

3. Los índices de nivel educativo e ingreso están tomados del Informe sobre el estado de la nación, publicado por el PNUD en 1999. El o la interesada puede consultar allí la metodología seguida para su construcción. 
toria política salvadoreña reciente constituyeron dos de los departamentos más conflictivos del país. Es posible pensar en una especie de tradición participativa como explicación plausible. La tradición de lucha de La Paz, que se remonta hasta la época de Anastasio Aquino, en el siglo XIX, y la tradición de resistencia del oriente, del Cerro de Guazapa, en Cuscatlán, apoyarían una explicación histórico-política de la participación electoral en El Salvador. Por el momento, no puedo profundizar más en este sentido y nada más dejo apuntadas estas observaciones a las cuales habría que añadir, para el caso de Chalatenango, el proceso participativo actual en torno al Comité Ambiental de Chalatenango $(\mathrm{CACH})^{4}$.

\subsection{Breve análisis diacrónico de la participa- ción}

¿Cuál ha sido la evolución de la participación electoral por circunscripciones desde 1994? ¿Es posible señalar algún tipo de tendencia? ¿Las circunscripciones que en el 2000 fueron las más participativas, lo han sido siempre desde 1994?
Para responder este tipo de interrogantes he elaborado el Cuadro 4, que se refiere a las elecciones parlamentarias. Las afirmaciones que se desprenden de su análisis deben considerarse con carácter tentativo, puesto que tres procesos electorales no son suficientes para hablar de tendencias claramente definidas. Habrá que esperar unos dos o tres procesos electorales más.

Antes de comentar el Cuadro 4, vale la pena hacer la siguiente aclaración. Para calcular la participación he tomado como base el total de votos válidos y los electores en el padrón correspondiente a cada año. En la medida en que éste no está del todo depurado, la cantidad de electores puede estar "inflada", influyendo en el cálculo de la participación. Con menos electores en el padrón y la misma cantidad de votos válidos para cada año, el nivel de participación sería mayor. Sin embargo, dado que lo que persigo es identificar algún tipo de tendencia a lo largo del tiempo, este efecto de "inflación" del padrón lo consideraré como que afectara a los tres años por igual ${ }^{5}$.

Cuadro 3

Participación electoral, educación e ingreso

\begin{tabular}{lccc}
\hline Circunscripción & $\begin{array}{c}\text { Participación } \\
\text { electoral (\%) }\end{array}$ & $\begin{array}{c}\text { Indice nivel } \\
\text { educativo }\end{array}$ & $\begin{array}{c}\text { Indice de } \\
\text { ingreso }\end{array}$ \\
\hline San Salvador & 38.4 & 0.843 & 0.564 \\
Santa Ana & 34.4 & 0.688 & 0.314 \\
San Miguel & 36.1 & 0.707 & 0.311 \\
La Libertad & 39.3 & 0.739 & 0.444 \\
Usulután & 38.4 & 0.669 & 0.247 \\
Sonsonate & 38.7 & 0.696 & 0.297 \\
La Unión & 34.5 & 0.576 & 0.143 \\
La Paz & 41.9 & 0.719 & 0.258 \\
Chalatenango & 47.1 & 0.681 & 0.181 \\
Cuscatlán & 40.5 & 0.736 & 0.271 \\
Ahuachapán & 37.7 & 0.691 & 0.266 \\
Morazán & 43.9 & 0.556 & 0.155 \\
San Vicente & 39.2 & 0.684 & 0.213 \\
Cabañas & 36.8 & 0.596 & 0.167 \\
\hline Nacional & 38.5 & 0.735 & 0.367 \\
\hline
\end{tabular}

Fuentes: Índice de nivel educativo e índice de ingreso, en PNUD (1997); Participación electoral, en Cuadro 1.

4. Sobre éste puede consultarse CACH-PROCHALATE (1999) y Enríquez, Alberto et al. (1997).

5. Esto no sería del todo cierto a menos que la "inflación" del padrón sea proporcionalmente la misma para cada año. Sin embargo, para el objetivo que persigo aquí, el procedimiento explicitado permite una aceptable aproximación para detectar tendencias. 
En general, es posible sostener una disminución constante en la participación electoral más pronunciada entre 1994-1997 que entre 19972000. También llama la atención que las cuatro circunscripciones más participativas en el 2000 también lo fueron en 1997, año en el que hay que incluir los casos de La Libertad y San Vicente. Sin embargo, lo más sobresaliente reside en la participación registrada en Chalatenango en los tres años electorales. Chalate ha sido el departamento más participativo en todo el período considerado.

\section{Cuadro 4}

Evolución de la participación electoral por circunscripciones, 1994-2000

(En porcentajes)

\begin{tabular}{llcl}
\hline Circunscripción & \multicolumn{3}{c}{ Participación } \\
\cline { 2 - 4 } & 1994 & 1997 & 2000 \\
\hline San Salvador & 54.5 & 39.2 & 38.4 \\
Santa Ana & 55.3 & 39.6 & 34.4 \\
San Miguel & 45.4 & 36.3 & 36.1 \\
La Libertad & 57.0 & 40.4 & 39.3 \\
Usulután & 49.7 & 36.3 & 38.4 \\
Sonsonale & 56.5 & 39.1 & 38.7 \\
La Unión & 45.7 & 33.7 & 34.5 \\
La Paz & 55.3 & 41.8 & 41.9 \\
Chalatenango & 57.3 & 45.4 & 47.1 \\
Cuscallán & 55.6 & 41.5 & 40.5 \\
Ahuachapán & 50.7 & 38.5 & 37.7 \\
Morazán & 49.6 & 40.8 & 43.9 \\
San Vicente & 57.6 & 44.0 & 39.2 \\
Cabañas & 48.8 & 34.3 & 36.8 \\
\hline Promedio & 53.5 & 39.2 & 38.5 \\
\hline
\end{tabular}

Fuentes: elaboración propia, según resultados oficiales.

\section{Partidos}

\section{1. ¿Bipartidismo o multipartidismo?}

Durante la campaña electoral, varios "analistas" hablaron de la existencia de un bipartidismo en El Salvador. Probablemente la fuerza electoral y parlamentaria que los partidos ARENA y FMLN han alcanzado desde 1994 motivaba a dichos "analistas" a referirse en esos términos". Sin embargo, tal y como lo he mostrado en otro trabajo (Artiga-González, 1999a), ni la concentración del voto y de los escaños en los dos primeros partidos, ni el número efectivo de partidos, en tanto índices para medir y comparar con otros sistemas de partidos, permitirían sostener empíricamente la "hipótesis" del bipartidismo? . Las elecciones del pasado 12 de marzo proporcionan otros datos contra esta hipótesis. Antes de presentarlos, debo hacer unas aclaraciones que considero necesarias, dado que estamos tratando con procesos electorales simultáneos.

En primer lugar, en El Salvador hay cuatro tipos de elecciones: presidenciales, legislativas, de concejos municipales y de diputados al Parlamento Centroamericano. En la medida en que esta última elección está supeditada a las votaciones para Asamblea Legislativa, no la consideraré en este análisis.

En segundo lugar, dada la forma presidencialista de nuestro gobierno, las elecciones presidenciales son las de mayor relevancia para el sistema de partidos. Pero, dado que la simultaneidad entre éstas y los otros dos tipos de elecciones ocurre sólo cada quince años y bajo sistemas electorales diferentes, es posible la existencia de dos lógicas de competición y de relaciones interpartidarias, que permiten hablar de dos sistemas de partidos.

En tercer lugar, la simultaneidad de elecciones parlamentarias y de concejos municipales se refiere a la hora, fecha, distribución de centros de votación y de electores en el respectivo padrón electoral. El resto de los elementos del sistema electoral (especialmente el tamaño y la distribución de las circunscripciones y la fórmula electoral) son tan disímiles que permiten la posibilidad de dos lógicas de competencia y votación y de relaciones interpartidistas diferentes. ¿Hasta qué punto es

6. En 1994, ARENA obtuvo con un 45 por ciento de votos el 46.4 por ciento de escaños; mientras que en 1997 obtuvo 33.3 por ciento de escaños con un 35.4 por ciento de los votos válidos. El FMLN obtuvo, en 1994, 21.4 por ciento de votos y 25.0 por ciento de escaños; en tanto que en 1997 alcanzó el 33.0 por ciento de los votos y 32.1 por ciento de escaños.

7. En otro trabajo (Artiga-González, 1996a) he mostrado que, atendiendo a ciertas propiedades funcionales propuestas por Sartori (1992) para los diferentes tipos de sistemas de partidos, el salvadoreño corresponde más bien al pluralismo polarizado. 
adecuado hablar en estas condiciones institucionales de un solo sistema de partidos para estas dos elecciones? Tomar en cuenta que las elecciones parlamentarias son elecciones de ámbito nacional (se elige un solo parlamento para todo el pais), mientras que las elecciones de concejos municipales son de ámbito local (se eligen autoridades para los 262 municipios que tiene el territorio nacional).

Con estas consideraciones creo que hablar de bipartidismo, sin más, es sumamente simplista. Aunque no debe causar extrañeza, pues, en el fondo, dicha impresión es parte de los efectos que el presidencialismo genera en las percepciones de la población incluyendo, por supuesto, a los llamados "analistas". $\mathrm{Si}$ hemos de ser rigurosos en el análisis científico, deberíamos considerar que para cada tipo de elección y para cada circunscripción electoral tenemos un sistema de partidos específico. Ignorar este detalle contribuye a pasar por alto la posibilidad de una reforma electoral, que permita la existencia de partidos políticos departamentales o municipales. No sabemos si una reforma en este sentido mejoraria la calidad de la representación política y si disminuiría su monopolio por parte de los partidos más grandes a nivel nacional. Pero como probabilidad allí está. De paso se podría favorecer que la disputa y el ejercicio del poder local tuvieran mayor autonomía respecto a la lucha y al ejercicio del poder en la capital, sede de los órganos de poder nacional.

\subsubsection{El formato partidista en las elecciones pariamentarias}

El Cuadro 5 muestra los porcentajes de votos y escaños de los partidos y/o coaliciones para el pe-

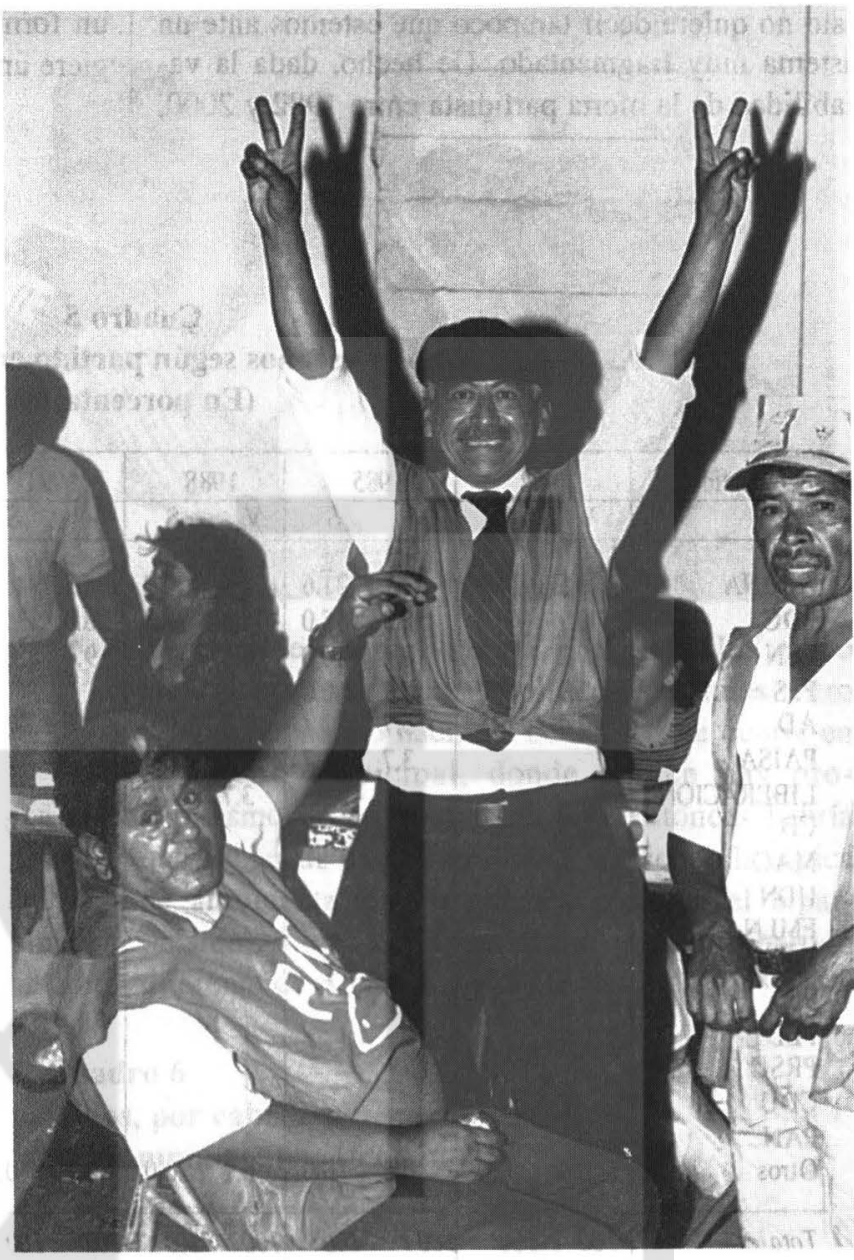

ríodo 1982-2000. Solamente he considerado a los partidos que para cada año electoral alcanzaron escaños. El cuadro también presenta el índice de Laakso y Taagepera (1979) para medir el número efectivo de partidos (electorales y parlamentarios), que en la ciencia política comparada se utiliza para definir el formato de los sistemas de partidos ${ }^{\sharp}$. Salvo para el año de 1985 , el número efectivo de partidos siempre ha estado arriba del valor de tres. Por tanto, en El Salvador, a nivel de elecciones parlamentarias, la lógica del sistema de partidos corresponde al multipartidismo, sin lugar a dudas.

8. Si se utilizan los votos para el cálculo, el número efectivo de partidos (electorales) se expresa mediante la fórmula $\mathrm{Nv}=1 /\left(\Sigma \mathrm{Vi}^{2}\right)$, donde $\mathrm{Vi}$ es la proporción de votos del i-ésimo partido. Y si se utilizan los escaños para el respectivo cálculo, el número efectivo de partidos (parlamentarios) es $\mathrm{Ns}=1 /\left(\sum \mathrm{Si}^{2}\right)$, donde $\mathrm{Si}$ es la proporción de escaños del i-ésimo partido. 
Esto no quiere decir tampoco que estemos ante un sistema muy fragmentado. De hecho, dada la variabilidad de la oferta partidista entre 1982 y 2000 , un formato promedio ligeramente arriba del 3 sugiere un sistema tripolar .

\section{Cuadro 5}

Votos y escaños según partido entre 1982-2000

(En porcentajes)

\begin{tabular}{|c|c|c|c|c|c|c|c|c|c|c|c|c|c|c|}
\hline \multirow[t]{2}{*}{ Partido } & \multicolumn{2}{|c|}{1982} & \multicolumn{2}{|c|}{1985} & \multicolumn{2}{|c|}{1988} & \multicolumn{2}{|c|}{1991} & \multicolumn{2}{|c|}{1994} & \multicolumn{2}{|c|}{1997} & \multicolumn{2}{|c|}{2000} \\
\hline & V & $S$ & V & S & $\mathrm{V}$ & $\mathbf{S}$ & V & $\bar{S}$ & $\mathrm{~V}$ & $\mathbf{S}$ & V & S & V & $\mathbf{S}$ \\
\hline ARENA & 29.5 & 31.7 & 29.7 & 21.6 & 48.0 & 50.0 & \begin{tabular}{|l|}
44.3 \\
\end{tabular} & 46.4 & 45.0 & 46.4 & 35.4 & 33.3 & 36.0 & 34.5 \\
\hline PDC & 40.1 & 40.0 & 52.3 & 55.0 & 35.2 & 38.3 & 28.0 & 31.0 & 17.9 & 21.4 & 8.4 & 10.7 & 7.2 & 6.0 \\
\hline PCN & 19.2 & 23.3 & 8.4 & 20.0 & 8.6 & 11.7 & 9.0 & 10.7 & 6.2 & 4.8 & 8.7 & 13.1 & 8.8 & 16.6 \\
\hline PPS & 2.9 & 1.7 & 1.7 & 0.0 & & & & & & & & & & \\
\hline$A D$ & 7.4 & & 3.7 & 1.7 & 1.7 & 0.0 & & & & & & & & \\
\hline PAISA & & & 3.7 & 1.7 & 2.1 & 0.0 & & & & & & & & \\
\hline LIBERACION & & & & & 3.7 & 0.0 & & & & & & & & \\
\hline & & & & & & & 12.1 & 9.5 & 4.4 & 1.2 & 3.5 & 2.4 & & \\
\hline MAC & & & & & & & 3.2 & 1.2 & & & & & & \\
\hline UDN & & & & & & & 2.7 & & & & & & & \\
\hline $\begin{array}{l}\text { FMLN } \\
\text { MU }\end{array}$ & & & & & & & & & 21.4 & 25.0 & 33.0 & 32.1 & 35.2 & 36.9 \\
\hline MU & & & & & & & & & 2.5 & 1.2 & 2.3 & 1.2 & & \\
\hline & & & & & & & & & & & 1.2 & & & \\
\hline PRSC & & & & & & & & & & & $\begin{array}{l}3.2 \\
3.6\end{array}$ & & & \\
\hline $\mathrm{CDU}$ & & & & & & & & & & & & & 5.4 & 3.6 \\
\hline PAN & & & & & & & & & & & & & 3.7 & 2.4 \\
\hline Otros & 0.4 & 0.0 & 0.5 & 0.0 & 0.7 & 0.0 & 0.7 & 0.0 & 2.6 & 0.0 & 0.7 & 0.0 & 3.7 & 0.0 \\
\hline Totales & 100 & 100 & 100 & 100 & 100 & 100 & 100 & 100 & 100 & 100 & 100 & 100 & 100 & 100 \\
\hline $\mathrm{Nv}$ & & .4 & 2. & & & .7 & & 3 & & 3.5 & & 3.9 & & 3.7 \\
\hline Ns & ( & & 2. & & &. .4 & & .0 & & 3.1 & & 4.1 & & 3.5 \\
\hline
\end{tabular}

Nv = Número efectivo de partidos electorales.

Ns = Número efectivo de partidos parlamentarios.

Fuente: elaboración propia sobre resultados oficiales.

9. No debe asimilarse lo tripolar con un esquema izquierda-centro-derecha. Por ejemplo, el sistema de partidos hondureño es un bipartidismo, pero los dos partidos principales están a la derecha. El caso salvadoreño es un caso tripolar, donde sí están definidos dos polos extremos (a la izquierda y a la derecha) y el tercero tiene un carácter fluido. En otro lugar he tipificado al sistema de partidos salvadoreño como sistema fluido (ArtigaGonzález, 1998). 
¿Qué ocurre en las elecciones municipales? Una análisis exhaustivo supondría examinar qué ocurre en los 262 municipios de la república, considerando a cada municipio tan autónomo del resto como para pensar en la existencia de 262 sistemas de partidos. ¡Ojo, no es una locura plantear las cosas así! Otra cosa es que no estemos acostumbrados a semejante análisis. Dado que no cuento con el espacio necesario para esta tarea en estos momentos, me limitaré al análisis de lo ocurrido en las catorce cabe-

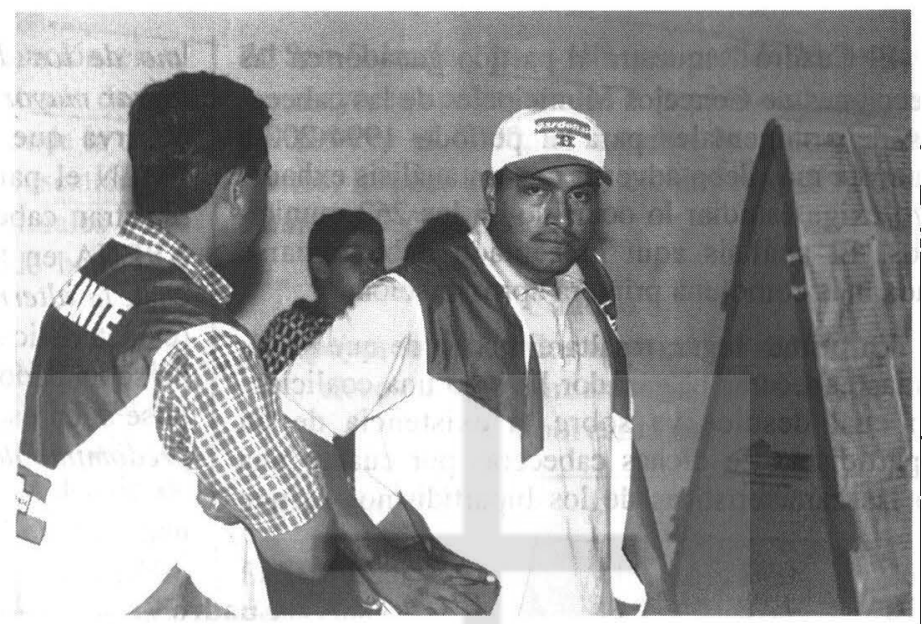
ceras departamentales como primera aproximación. Temporalmente me fijaré en el período 1994-2000 (tres elecciones) para ver en perspectiva lo ocurrido el pasado 12 de marzo.

De acuerdo con la información mostrada en el Cuadro 6, tampoco puede hablarse de bipartidismo en el caso de las elecciones de concejos municipales. ¿De dónde, entonces, surge la idea del bipartidismo salvadoreño? Creo que del protagonismo

nacional de los partidos ARENA y FMLN como las dos primeras fuerzas político-electorales. Pero ese protagonismo nacional oculta lo que ocurre en el ámbito municipal, donde o bien hay protagonismo de un solo partido (y entonces habría que hablar de monopartidismo, siguiendo la lógica de argumentación de quienes postulan el bipartidismo), o bien el protagonismo es de otro partido diferente de ARENA y el FMLN.

Cuadro 6

Formato del sistema de partidos, por cabecera departamental, en elecciones de concejos municipales, 1994-2000

\begin{tabular}{llll}
\hline Cabeceras & 1994 & 1997 & 2000 \\
\hline San Salvador & 3.1 & 3.1 & 2.1 \\
Santa Ana & 3.4 & 3.3 & 3.5 \\
San Miguel & 3.6 & 3.9 & 3.5 \\
Nueva San Salvador & 3.0 & 3.3 & 2.8 \\
Usulután & 3.5 & 4.6 & 3.3 \\
Sonsonate & 3.9 & 3.7 & 3.1 \\
La Unión & 3.1 & 3.6 & 2.6 \\
Zacatecoluca & 4.2 & 3.6 & 3.0 \\
Chalatenango & 3.4 & 3.2 & 2.4 \\
Cojutepeque & 2.8 & 3.7 & 4.2 \\
Ahuachapán & 4.1 & 4.1 & 3.6 \\
San Francisco Gotera & 3.8 & 4.6 & 3.5 \\
San Vicente & 3.4 & 3.0 & 3.0 \\
Sensuntepeque & 3.1 & 3.2 & 4.7 \\
\hline
\end{tabular}

Fuente: elaboración propia sobre resultados oficiales. 
El Cuadro 7 muestra al partido ganador en las elecciones de Concejos Municipales de las cabeceras departamentales para el período 1994-2000. Una vez más debo advertir que un análisis exhaustivo exige estudiar lo ocurrido en los 262 municipios. El análisis aquí presentado debe tomarse nada más como una primera aproximación.

En primer lugar, resalta el hecho de que en algunas cabeceras el ganador ha sido una coalición. Lo cual desdice ya sobre la existencia de un bipartidismo en dichas cabeceras por cuanto una de las características de los bipartidismos es que uno de los dos partidos relevantes es capaz de lograr mayoria por sí solo. En segundo lugar, se observa que no siempre ha sido ARENA o el FMLN el partido ganador. $Y$, en tercer lugar, se registran cabeceras en las que el ganador ha sido ARENA en los tres procesos electorales. Con lo cual, la alternancia en el poder en esas cabeceras (característica también de los bipartidismos) aún no se ha dado. El analista más bien podría preguntarse si en esos casos no habría un pluralismo con predominio de uno de los partidos (Sartori, 1992).

\section{Cuadro 7}

Control de concejos municipales, según partido, 1994-2000

\begin{tabular}{lccc}
\hline \multirow{2}{*}{ Municipio } & \multicolumn{3}{c}{ Partido o coalición de gobierno } \\
\cline { 2 - 4 } & 1994 & 1997 & 2000 \\
\hline San Salvador & ARENA & FMLN-CD-MU & FMLN-USC \\
Santa Ana & ARENA & FMLN-CD-MU & FMLN-PDC \\
San Miguel & ARENA & ARENA & PDC \\
Nueva San Salvador & ARENA & FMLN & FMLN \\
Usulután & ARENA & ARENA & PCN \\
Sonsonate & ARENA & FMLN-CD & FMLN \\
La Unión & PDC & PDC & ARENA \\
Zacatecoluca & ARENA & FMLN & FMLN \\
Chalatenango & ARENA & FMLN & FMLN \\
Cojutepeque & ARENA & PRSC & ARENA \\
Ahuachapán & ARENA & ARENA & FMLN \\
San Francisco Gotera & ARENA & ARENA & PD-FMLN-CD \\
San Vicente & ARENA & ARENA & ARENA \\
Sensuntepeque & ARENA & ARENA & ARENA \\
\hline
\end{tabular}

Fuente: elaboración propia sobre resultados oficiales.

\subsection{Análisis de "feudos"}

En este apartado haré una revisión de la evolución de los apoyos electorales de los partidos que en las elecciones del 12 de marzo obtuvieron escaños legislativos ${ }^{10}$. La unidad de observación corresponde a las catorce circunscripciones departamentales (para las elecciones parlamentarias) y a las catorce cabeceras departamentales (como pri- mera aproximación al análisis del comportamiento electoral municipal o local).

\subsubsection{Elecciones legislativas}

A juzgar por lo que se observa en el Cuadro 8, el partido ARENA ha perdido apoyo sustancial en prácticamente todos los departamentos de la república, si se toma como punto de partida el resulta-

10. Por razones de comparación excluyo al PAN, puesto que en el 2000 es la primera vez que participa y obtiene escaños legislativos. 
do de 1994. Ahora bien, esta disminución en el caudal de votos arenero no se ha registrado de forma homogénea. Hay departamentos donde la caída ha sido más pronunciada como en Cuscatlán, en donde ha perdido 16.9 puntos porcentuales entre 1994 y el 2000, y Ahuachapán, donde bajó 13.9 puntos entre 1994 y 1997.

Por otra parte, también puede observarse que hay departamentos en los cuales ARENA recuperó fuerza electoral en el 2000 , luego de una caída en 1997, aunque su recuperación está por debajo de los niveles alcanzados en 1994. Es el caso de San Salvador, La Libertad, La Unión, Cuscatlán, Ahuachapán y Cabañas. En este último departamento, ARENA se ha mantenido, de todas formas, por encima de los 40 puntos porcentuales en todo el período. Por otro lado, en los casos de Santa Ana, San Miguel, Chalatenango, Usulután, Sonsonate y La Paz, el partido ARENA, pese a seguir en descenso, ha rondado los dos puntos porcentuales entre 1997 y el 2000 . Finalmente, si tomamos en cuenta que a nivel nacional el caudal promedio de votos legislativos en el 2000 es del 36.0 por ciento, resaltan los casos de La Libertad y Cabañas, pues allí se registraron caudales de 5.7 y 7.9 puntos por arriba de dicho promedio. En general, pues, después del descenso pronunciado entre 1994 y 1997, ARENA parece sostener porcentualmente las preferencias de un poco más del tercio del electorado salvadoreño. De hecho, el caudal de votos en el 2000 ha sido ligeramente superior al de 1997.

¿Cuál ha sido la suerte electoral del partido FMLN a lo largo de los tres procesos electorales que estamos considerando? El Cuadro 9 nos servirá como guía para el análisis. En primer lugar, hay que señalar el sostenido crecimiento de su caudal electoral. Salvo el caso de Santa Ana, en el 2000, donde se registra un pequeño descenso de 0.5 puntos porcentuales, y Morazán, en 1997, donde también se registra un decremento de 1.7 puntos respecto a 1994, el FMLN ha ido ganando terreno en las preferencias electorales en todos los departamentos del país. Sobresale el caso de San Salvador, pues allí este partido ha logrado sus mayores apoyos en los tres años. Además, entre 1994 y el 2000 , el ascenso ha sido de 15.3 puntos, lo cual implica un crecimiento del 52 por ciento respecto a 1994.

\section{Cuadro 8}

Apoyos territoriales al partido ARENA en elecciones parlamentarias, 1994-2000 (En porcentajes)

\begin{tabular}{llll}
\hline Departamento & 1994 & 1997 & 2000 \\
\hline San Salvador & 44.8 & 34.0 & 36.1 \\
Santa Ana & 43.9 & 37.5 & 37.5 \\
San Miguel & 44.6 & 34.0 & 31.6 \\
La Libentad & 47.9 & 38.9 & 41.7 \\
Usulután & 44.1 & 34.5 & 33.9 \\
Sonsonate & 43.1 & 33.4 & 31.0 \\
La Unión & 45.6 & 33.6 & 39.6 \\
La Paz & 46.6 & 34.4 & 33.2 \\
Chalatenango & 45.1 & 41.0 & 39.3 \\
Cuscatlán & 52.4 & 33.0 & 35.5 \\
Ahuachapán & 41.1 & 27.2 & 34.5 \\
Morazán & 39.5 & 35.0 & 31.0 \\
San Vicente & 43.2 & 39.1 & 36.5 \\
Cabañas & 49.6 & 40.0 & 43.9 \\
\hline
\end{tabular}

Fuente: elaboración propia, según resultados oficiales.

Por otra parte, este crecimiento refleja un comportamiento más homogéneo entre los efemelenistas, en comparación con los areneros. Mientras los apoyos territoriales de ARENA bajan, suben o se mantienen, todas las bases territoriales del 
FMLN se expanden. Sin embargo, esta expansión ha ocurrido a ritmos variables. Hay casos como San Miguel, Sonsonate, La Unión, Ahuachapán y Cabañas en los cuales el crecimiento ha sido del 100 por ciento o más, entre 1994 y el 2000 . Por otro lado, es llamativo el caso de La Unión, pues aunque su crecimiento haya sido del 128 por ciento, el nivel absoluto en el 2000 es casi un tercio del registrado en San Salvador en este mismo año. De hecho, en La Unión es donde menos bases temitoriales tiene el FMLN.

En general, el crecimiento promedio del FMLN a nivel nacional, en el 2000 , ha sido apenas de 2.2 puntos porcentuales respecto a 1997 . Un poco más que el crecimiento territorial del partido ARENA, pero aglutinando también cerca de un tercio del electorado salvadoreño. Vale la pena detenernos aquí para introducir otro comentario sobre el supuesto bipartidismo salvadoreño. El énfasis que algunos analistas hacen en el comportamiento de los diputados en la Asamblea Legislativa los lleva a no fijarse en el comportamiento electoral de las bases de los partidos. Los partidos no son sólo los diputados, sino también sus bases electorales. A este nivel, ARENA y el FMLN concentran un poco más de los dos tercios del electorado. El resto se lo reparten los otros partidos, lo cual nos deja otra vez en el ámbito del multipartidismo.

\section{Cuadro 9}

Apoyos territoriales al partido FMLN en elecciones parlamentarias, 1994-2000 (En porcentajes)

\begin{tabular}{lccc}
\hline Departamento & 1994 & 1997 & 2000 \\
\hline San Salvador & 29.5 & 41.6 & 44.8 \\
Santa Ana & 21.5 & 31.6 & 31.1 \\
San Miguel & 15.0 & 27.4 & 30.6 \\
La Libertad & 21.9 & 34.3 & 36.8 \\
Usulután & 15.9 & 28.7 & 35.9 \\
Sonsonate & 16.7 & 31.9 & 33.3 \\
La Unión & 6.8 & 11.7 & 15.5 \\
La Paz & 16.0 & 31.1 & 27.6 \\
Chalatenango & 18.9 & 29.1 & 31.4 \\
Cuscatlán & 18.3 & 27.7 & 29.1 \\
Ahuachapán & 16.0 & 26.1 & 31.5 \\
Morazán & 15.8 & 14.1 & 23.7 \\
San Vicente & 22.7 & 38.5 & 38.6 \\
Cabañas & 12.1 & 22.6 & 23.2 \\
\hline
\end{tabular}

Fuente: elaboración propia, según resultados oficiales.
¿Cómo se distribuye territorialmente el "otro tercio" del electorado salvadoreño? Por razones de espacio me centraré en el análisis de los apoyos territoriales del PCN y del PDC. Estos partidos no necesariamente concentran totalmente ese "otro tercio", pero curiosamente son los partidos más viejos de todo el espectro partidista salvadoreño. Ambos proceden de la década de los años sesenta del siglo pasado y tienen, por tanto, cerca de los cuarenta años de existencia. El hecho de que tengan una presencia parlamentaria del 22 por ciento, en conjunto, indica la posibilidad de la existencia de identidades partidistas resistentes al paso del tiempo. Este hecho es digno de tomarlo en cuenta, sobre todo porque podría expresar una lealtad de su electorado, a quien sus dirigentes deberían escuchar más. Probablemente esto es algo que no ha ocurrido y es posible que esté en la base del continuo descenso del apoyo al PDC, desde su máximo histórico en 1985.

Cuadro 10

Apoyos territoriales al Partido de Conciliación Nacional en elecciones parlamentarias, 1994-2000 (En porcentajes)

\begin{tabular}{lrrr}
\hline Departamento & 1994 & 1997 & 2000 \\
\hline San Salvador & 3.9 & 4.7 & 3.2 \\
Santa Ana & 5.2 & 8.5 & 6.4 \\
San Miguel & 6.0 & 10.5 & 6.9 \\
La Libertad & 4.4 & 7.2 & 5.2 \\
Usulután & 5.9 & 11.8 & 15.6 \\
Sonsonate & 7.6 & 9.7 & 9.5 \\
La Unión & 7.3 & 17.9 & 24.2 \\
La Paz & 10.7 & 16.8 & 18.9 \\
Chalatenango & 7.5 & 5.1 & 9.1 \\
Cuscatlán & 8.3 & 11.2 & 13.5 \\
Ahuachapán & 13.0 & 8 & 11.2 \\
Morazán & 11.3 & 8.3 & 20.3 \\
San Vicente & 4.2 & 7.4 & 10.1 \\
Cabañas & 13.4 & 23.5 & 15.2 \\
\hline
\end{tabular}

Fuente: elaboración propia, según resultados oficiales.

El PCN ha resistido el cambio de régimen y pareciera incluso tener un electorado total estable, desde 1985, entre el 8 y 9 por ciento (ver Cuadro 5). ¿Qué tan exacta es esta apreciación? Según el 
Cuadro 10, los apoyos territoriales del Partido de Conciliación Nacional están distribuidos de forma heterogénea a lo largo y ancho del país, así como en el transcurso del tiempo. Se podría pensar en un electorado volátil, inestable, sometido a vaivenes coyunturales. Esto puede notarse por el movimiento de ascenso y descenso (o a la inversa) de las preferencias pecenistas en los tres años electorales, en donde 1997 es el pivote o el punto de inflexión. De los catorce departamentos, este movimiento de sube y baja (o a la inversa) se observa en nueve. En los cinco departamentos restantes, el movimiento ha sido de ascenso continuo. Sobresale La Unión, donde el PCN ha subido 16.9 puntos desde 1994. San Salvador es el departamento donde menos apoyo tiene este partido, que es de apenas un 3.2 por ciento. Entre San Salvador y La Unión, el rango de variación de las preferencias pecenistas fue de 21 puntos porcentuales en el 2000. Así que la aparente estabilidad del 8-9 por ciento de votos del PCN, a nivel nacional, oculta su inestable y heterogénea distribución territorial departamental. El porcentaje nacional de votos que alcanzó el $\mathrm{PCN}$ en las recientes elecciones, y que lo ubica como tercera fuerza electoral, no deja ver que, por ejemplo, en La Unión, el PCN resultó en segunda posición con 8.5 puntos porcentuales arriba del FMLN. Análogamente, en San Miguel, el PCN no es la tercera fuerza, puesto que es superado por el PDC por 15.1 puntos. Finalmente, el departamento de mayor estabilidad en cuanto al apoyo al PCN ha sido Sonsonate con una pequeña variación de 1.9 desde 1994 hasta el 2000 . Una variación que se reduce a 0.2 en las dos últimas elecciones.

Los apoyos territoriales del PDC han seguido una trayectoria más homogénea que los pecenistas. Salvo los casos de San Miguel y Cabañas, en todos los departamentos se ha registrado un descenso continuo del apoyo al PDC, desde 1994. Este descenso, que se remonta ciertamente a 1988, cuando el PDC perdió la mayoría en la Asamblea Legislativa, parece no tener fin, pues, como se observa en el Cuadro 11, si en 1994 el PDC tenía apoyos superiores al 20 por ciento de los votos válidos en diez de los catorce departamentos, en el 2000 , tal nivel de apoyo se registró únicamente en
San Miguel. De igual forma: si en 1994 todos los departamentos registraban apoyos pedecistas por arriba del 10 por ciento, en el 2000 ese apoyo solamente se encuentra en tres departamentos, o sea que, en once de ellos, las preferencias pedecistas no llegan ni al 10 por ciento de los votos. El mínimo apoyo se registró en San Salvador y el máximo en San Miguel con un rango de variación, entre estos departamentos, de un 18.7 por ciento. Vale agregar que San Salvador fue el lugar de menor fuerza electoral del PDC en todo el período. Se trata de un resultado paradójico si se recuerda que, en los años sesenta y setenta del siglo pasado, San Salvador fue justamente el bastión de la oposición, encabezada por el Partido Demócrata Cristiano.

\section{Cuadro 11}

Apoyos territoriales al Partido Demócrata Cristiano en elecciones parlamentarias, 1994-2000

(En porcentajes)

\begin{tabular}{lrrr}
\hline Departamento & 1994 & 1997 & 2000 \\
\hline San Salvador & 11.4 & 3.7 & 3.2 \\
Santa Ana & 21.0 & 11.3 & 7.4 \\
San Miguel & 23.6 & 10.4 & 22.0 \\
La Libertad & 15.6 & 5.6 & 5.1 \\
Usulután & 21.1 & 10.6 & 6.2 \\
Sonsonate & 23.5 & 12.5 & 5.9 \\
La Unión & 30.1 & 20.2 & 14.6 \\
La Paz & 20.3 & 9.7 & 12.6 \\
Chalatenango & 22.2 & 7.8 & 5.8 \\
Cuscatlán & 12.4 & 3.9 & 3.8 \\
Ahuachapán & 24.0 & 14.7 & 8.4 \\
Morazán & 23.5 & 12.6 & 9.7 \\
San Vicente & 22.5 & 9.5 & 6.8 \\
Cabañas & 15.7 & 5.9 & 6.9 \\
\hline
\end{tabular}

Fuente: elaboración propia, según resultados oficiales.

\subsubsection{Elecciones para concejos municipales}

Antes de 1994, el partido ARENA venía ganando cada vez más municipios con cada vez menos votos (Artiga-González, 1996a). Los apoyos territoriales de los diferentes partidos, en 1997 y 
en el 2000, han modificado esta tendencia. De hecho, dos movimientos sobresalen. Por un lado, ARENA ha ido perdiendo alcaldias desde 1997. Por el otro, el FMLN, solo o en coalición, ha ido aumentando su control sobre gobiernos municipales. Si en 1994 el FMLN no resultó ganador en ninguna de las catorce cabeceras departamentales, en el 2000 logró ganar en ocho cabeceras. ARENA ha pasado de controlar trece cabeceras en 1994 a cinco en el 2000. El PDC logró el control de una cabecera en las tres elecciones, aunque no necesariamente se trata de la misma. De hecho, en 1994 y 1997 ganó La Unión, mientras que en el 2000 se agenció la alcaldía de San Miguel.

Los Cuadros 12, 13, 14 y 15 detallan la evolución del apoyo territorial a los partidos ARENA, FMLN, PCN y PDC, en las catorce cabeceras departamentales, en los tres años electorales que vengo considerando. En relación con el partido ARENA, pueden notarse dos movimientos en el transcurso del tiempo, desde 1994 hasta el 2000. Por un lado, en seis cabeceras departamentales, ARENA ha perdido votos de forma continua. Por otro lado, en las restantes ocho cabeceras se registró un movimiento de pérdida (1994-1997) y de recuperación (1997-2000). Ahora bien, este último movimiento de recuperación no devuelve el apoyo territorial arenero a los niveles registrados en 1994. Sin embargo, llama la atención el caso de La Unión, donde sí se registró un incremento, incluso por encima del nivel de 1994. ARENA alcanzó un $\mathbf{5 0 . 6}$ por ciento de los votos válidos en esta cabecera, 15.1 puntos porcentuales por arriba de lo que obtuvo en 1994. Semejante nivel de apoyo territorial únicamente se había registrado en Cojutepeque y Sensuntepeque, en 1994.

Por otra parte, una clasificación de las cabeceras departamentales, según el nivel de apoyo logrado por el partido, nos mostraría una distribución bastante homogénea. ARENA alcanza entre un 30 y $\mathbf{4 0}$ por ciento de las preferencias en nueve cabeceras, y entre un 40 y 50 por ciento en 2 cabeceras. Si tomamos en cuenta que estas dos cabeceras (Chalatenango y San Vicente) están por debajo del 42 por ciento, los dos grupos señalados podrían considerarse uno solo, con once cabeceras. El resto se distribuye así: una cabecera con más del 50 por ciento de los votos válidos y dos cabeceras por debajo del 30 por ciento. El máximo de apoyo está en La Unión y el mínimo en San Miguel, con un rango de variación de 25.9 puntos.

\section{Cuadro 12}

Apoyos territoriales al partido ARENA en elecciones municipales, 1994-2000 (En porcentajes)

\begin{tabular}{llll}
\hline Cabecera & 1994 & 1997 & 2000 \\
\hline San Salvador & 48.4 & 40.7 & 39.0 \\
Santa Ana & 45.4 & 35.9 & 33.3 \\
San Miguel & 43.6 & 35.2 & 24.7 \\
Nueva San Salvador & 49.6 & 38.4 & 37.6 \\
Usulután & 47.6 & 33.1 & 34.4 \\
Sonsonate & 41.2 & 36.7 & 37.5 \\
La Unión & 35.5 & 27.7 & 50.6 \\
Zacatecoluca & 36.7 & 26.0 & 29.7 \\
Chalatenango & 44.9 & 36.4 & 41.9 \\
Cojutepeque & 50.5 & 22.8 & 35.2 \\
Ahuachapán & 38.6 & 29.8 & 34.4 \\
San Francisco Gotera & 38.4 & 32.8 & 34.5 \\
San Vicente & 45.5 & 44.0 & 41.8 \\
Sensuntepeque & 53.8 & 45.8 & 32.9 \\
\hline
\end{tabular}

Fuente: elaboración propia, según resultados oficiales.

En el caso de los apoyos territoriales del FMLN, los movimientos que se registran siguen trayectorias exactamente contrarias a las señaladas para el caso de ARENA. Según el Cuadro 13, en diez cabeceras departamentales el FMLN ha tenido un crecimiento constante, mientras que en las cuatro restantes, el movimiento ha sido de sube y baja; pero sólo en un caso (La Unión) tal descenso significó un nivel de apoyo territorial por debajo del nivel alcanzado en 1994. Si bien hay cierta homogeneidad en el transcurso del tiempo, la distribución territorial de los votos efemelenistas es más heterogénea que la de los votos areneros. Si se establecen rangos de variación de 10 puntos porcentuales, las catorce cabeceras departamentales tienen que ser agrupadas en seis clases. 
Cuadro 13

Apoyos territoriales al partido FMLN en elecciones municipales, 1994-2000

(En porcentajes)

\begin{tabular}{lrrr}
\hline Cabecera & 1994 & 1997 & 2000 \\
\hline San Salvador & 24.2 & 39.3 & $53.3^{\prime \prime}$ \\
Santa Ana & 25.7 & 40.9 & $36.0^{\mathrm{b}}$ \\
San Miguel & 17.4 & 33.3 & 20.0 \\
Nueva San Salvador & 26.4 & 38.6 & 46.0 \\
Usulután & 10.7 & 23.5 & $19.7^{\circ}$ \\
Sonsonate & 17.6 & 34.1 & 41.8 \\
La Unión & 6.1 & 11.9 & 5.6 \\
Zacatecoluca & 22.4 & 42.5 & 47.8 \\
Chalatenango & 23.8 & 40.9 & 48.7 \\
Cojutepeque & 15.9 & 24.7 & 28.5 \\
Ahuachapán & 19.1 & 29.7 & 35.9 \\
San Francisco Gotera & 10.3 & 9.3 & $35.8^{d}$ \\
San Vicente & 23.8 & 34.7 & 38.6 \\
Sensuntepeque & 11.5 & 17.4 & 23.8 \\
\hline
\end{tabular}

amln-usc

"fmln-pde

c fmln-cdu

d fmln-cdu

Fuente: elaboración propia, según resultados oficiales.

Al igual que ARENA, el FMLN ha logrado una cabecera con un apoyo superior al 50 por ciento de los votos válidos (San Salvador), aunque no hay que pasar por alto el hecho de que, en este caso, los votos presentados en el cuadro se refieren a la coalición (FMLN-USC). En cuatro cabeceras, el FMLN alcanzó entre el 40 y 50 por ciento de los votos, y en otras cuatro, entre el 30 y 40 por ciento. En una cabecera aglutinó entre el 10 y el 20 por ciento de los votos, y en la restante no llegó ni al 10 por ciento. Esta última es La Unión, donde apenas tuvo un 5.6 por ciento de los votos; justamente en la cabecera donde ARENA obtuvo su máximo. El rango de variación de los votos efemelenistas, en las catorce cabeceras, resultó de 50.7 puntos porcentuales entre San Salvador y La Unión. Vale la pena agregar que los departamentos cuyas cabeceras son las mencionadas, también definieron el rango de variación de los apoyos territoriales en el caso de las elecciones parlamentarias. Finalmente, en tres de las cuatro cabeceras departamentales del oriente del país, el apoyo efemelenista fue menor respecto al resto de cabeceras del país.
De las catorce cabeceras que estoy considerando en este análisis, una fue ganada por el PCN (Usulután) y otra por el PDC (San Miguel). Esta casi nula eficacia de estos partidos se puede observar en los mínimos porcentajes de su apoyo territorial, mostrados en los Cuadros 14 y 15 . Sin embargo, en el caso del PCN, vale la pena fijarse en Usulután, pues allí este partido alcanzó su mayor cuota de apoyo territorial (37.1 por ciento). Nueve cabeceras registraron apoyos menores al 10 por ciento y, en las cuatro cabeceras restantes, el PCN osciló entre el 10 y 20 por ciento de los votos. El rango de variación fue muy amplio, pues osciló desde el 1.3 por ciento en San Salvador hasta el 37.1 por ciento en Usulután, dando un total de 35.8 puntos porcentuales. En cuanto a tendencias en la distribución de los apoyos territoriales en el transcurso del tiempo, el Cuadro 14 muestra una imagen bastante diversa con seis tipos de movimientos. En cuatro cabeceras (San Salvador, Sonsonate, Zacatecoluca y Chalatenango), el PCN ha ido perdiendo continuamente adeptos; en cinco (Santa Ana, San Miguel, La Unión, San Vicente y Sensuntepeque) ha tenido un movimiento de ascenso y descenso; en una (Nueva San Salvador) una trayectoria casi constante; en otra (Ahuachapán) un movimiento de descenso y ascenso; en dos (San Francisco Gotera y Usulután), la trayectoria ha sido de constante crecimiento (aunque en pequeño grado en la primera) y, finalmente, en Cojutepeque ha tenido un movimiento de estabilidad y crecimiento.

En el caso del Partido Demócrata Cristiano, pese a registrar un rango de variación territorial de 40.4 puntos, once de las catorce cabeceras se ubican por debajo del 10 por ciento de votos pedecistas; dos están entre el 30 y 40 por ciento (una de éstas en coalición con el FMLN) y una por arriba del 40 por ciento, justamente donde el PDC se adjudicó el control del gobierno municipal (San Miguel). En cuanto a las trayectorias de variación de los apoyos territoriales, en el transcurso del tiempo, los movimientos registrados en las distintas cabeceras son de cuatro tipos, aunque concentrados en dos. En primer lugar, seis cabeceras (San Salvador, Usulután, Sonsonate, La Unión, Zacatecoluca y Chalatenango) muestran movi- 
miento de constante descenso; en segundo lugar, otras seis cabeceras (Santa Ana, San Miguel, Nueva San Salvador, Cojutepeque, San Francisco Gotera y San Vicente) han tenido un movimiento de descenso y recuperación, resaltando el caso de San Miguel (donde el PDC se agenció la alcaldía) y Santa Ana, donde la recuperación se debió a su participación en una coalición con el FMLN. Los otros dos tipos de trayectorias se ubican en Ahuachapán —crecimiento-disminución- y Sensuntepeque -descenso y estabilización práctica-.

\section{Cuadro 14}

Apoyos territoriales al Partido de Conciliación Nacional en elecciones municipales, 1994-2000 (En porcentajes)

\begin{tabular}{lrrr}
\hline Cabecera & 1994 & 1997 & 2000 \\
\hline San Salvador & 8.2 & 2.7 & 1.3 \\
Santa Ana & 5.1 & 6.5 & 4.4 \\
San Miguel & 6.9 & 7.7 & 2.9 \\
Nueva San Salvador & 3.8 & 3.8 & 3.9 \\
Usulután & 6.5 & 17.7 & 37.1 \\
Sonsonate & 14.9 & 7.6 & 3.5 \\
La Unión & 3.6 & 12.5 & 5.1 \\
Zacatecoluca & 14.6 & 14.2 & 11.0 \\
Chalatenango & 7.9 & 5.5 & 2.2 \\
Cojutepeque & 5.4 & 5.4 & 9.9 \\
Ahuachapán & 17.6 & 8.7 & 16.5 \\
San Francisco Gotera & 15.4 & 16.0 & 18.5 \\
San Vicente & 8.5 & 9.7 & 7.0 \\
Sensuntepeque & 8.6 & 26.6 & 17.1 \\
& & & \\
\hline
\end{tabular}

Fuente: elaboración propia, según resultados oficiales.

Si quisiera resumir todos estos movimientos registrados en los apoyos territoriales de los cuatro partidos analizados, podría decir que corresponden a un estado de fluidez de los alineamientos electorales $^{11}$. Es decir, el sistema de partidos salvadoreno no termina de estructurarse. Una conclusión que refuerza el planteamiento de que la consolidación del régimen democrático salvadoreño todavía no se ha alcanzado.

\section{Cuadro 15}

Apoyos territoriales al Partido Demócrata

Cristiano en elecciones municipales, 1994-2000 (En porcentajes)

\begin{tabular}{lrrr}
\hline Cabecera & 1994 & 1997 & 2000 \\
\hline San Salvador & 13.4 & 2.6 & 2.1 \\
Santa Ana & 13.6 & 5.9 & $\ldots .$. \\
San Miguel & 22.8 & 7.6 & 42.5 \\
Nueva San Salvador & 8.3 & 3.5 & 4.9 \\
Usulután & 18.0 & 11.6 & $8.4^{n}$ \\
Sonsonate & 16.8 & 8.5 & 3.4 \\
La Unión & 44.4 & 40.8 & 35.3 \\
Zacatecoluca & 16.1 & 7.9 & 6.7 \\
Chalatenango & 15.9 & 3.5 & 2.3 \\
Cojutepeque & 15.8 & 1.9 & $8.5^{\circ}$ \\
Ahuachapán & 16.9 & 24.6 & 4.2 \\
San Francisco Gotera & 27.8 & 5.5 & 6.4 \\
San Vicente & 13.4 & 6.0 & 7.4 \\
Sensuntepeque & 12.8 & 5.4 & 5.5 \\
\hline
\end{tabular}

- fmln-pdc

"pan-pdc-usc

c pdc-usc

Fuente: elaboración propia, según resultados oficiales.

\section{La caja de Pandora}

Antes y después de las elecciones del 12 de marzo, se insistió en la necesidad de introducir reformas al sistema electoral para facilitar la participación ciudadana. Un tópico al respecto tiene que ver con el padrón electoral y las anomalías que éste presenta ("muertos" que votan, "vivos" que no aparecen, "migrantes" que ya no están en el país, nombres de ciudadanos que no corresponden con el número de camé, etc.). Sin desconocer la transcendencia que el padrón tiene para condicionar los resultados electorales, en esta parte me referiré a otros temas que los resultados "favorables" (según algunos analistas) al PCN sacaron a luz y debate público. Por cuestiones de espacio, restringiré el análisis a las elecciones parlamentarias dejando para otra ocasión lo relativo a las elecciones municipales.

11. En otro trabajo me he referido a la fluidez de la oferta partidista del sistema salvadoreño (Artiga-González, 1999b). El análisis hecho aquí, por el lado de la demanda, refuerza la tesis sostenida allí acerca del tipo fluido del sistema de partidos salvadoreño. 


\subsection{Proporcionalidad y desproporcionalidad}

Inmediatamente que se supo que el PCN había alcanzado 14 diputaciones, la atención se dirigió con fuerza hacia el sistema de reparto de los escaños. "Horrorizado" por tal resultado, el periodista Ricardo Vaquerano escribe un reportaje titulado: "PCN: menos votos pero más diputados" y sostiene que "la proporcionalidad es un cuento cuando el sistema de residuos permite a un partido formar su bancada con un promedio de votos mucho más bajo que el de sus adversarios" (Vaquerano, 2000, p. $12)^{12}$. El reportaje machaca una y otra vez que, gracias al sistema de residuos, al PCN le costaron menos votos sus diputados respecto del resto de partidos. Así, por ejemplo, resalta cómo por cada tres diputados del PCN, la Asamblea Legislativa 20002003 tendrá uno del CDU y otro del PAN y que "el partido que más desperdició votos fue el PAN".

El asombro lo ocasiona, pues, el resultado "inesperado" para el PCN. Pero, ies el sistema de residuos (como le llama Vaquerano) el responsa- ble? En otros trabajos he apuntado algunas fuentes de desproporcionalidad en nuestro sistema electoral. Ninguna de ellas apunta dramáticamente al sistema de residuos que en la Ciencia Política recibe el nombre de fórmula electoral. Es cierto que ésta produce efectos desproporcionales, pero no en la forma como parece sugerir Vaquerano ${ }^{13}$. Reiteradamente he mostrado que la causa mayor hay que buscarla en la distribución y la magnitud de las circunscripciones electorales (Artiga-González, $1993 ; 1996 b$ y $1997 b)$.

En primer lugar, está la mala distribución de diputados por cada departamento. Esto puede observarse claramente en el Cuadro 16 . Si seguimos la lógica de nuestro reportero, resulta que por cada 98063 habitantes o por cada 18910 votos válidos, debería designarse un diputado. Es evidente que esto no ocurre así. No se distribuyen los diputados según el total nacional de habitantes ${ }^{14}$, como tampoco se distribuyen los diputados según el total nacional de votos válidos que obtiene cada partido.

\section{Cuadro 16}

Distribución de diputados por departamento, según población y cociente electoral

\begin{tabular}{lrrrrrr}
\hline \multicolumn{1}{c}{ Departamento } & Población" & M" $^{\prime \prime}$ & $\begin{array}{r}\text { Razón de } \\
\text { represent. }\end{array}$ & $\begin{array}{c}\text { Votos } \\
\text { válidos }\end{array}$ & $\begin{array}{r}\text { Cociente } \\
\text { electoral }\end{array}$ \\
\hline San Salvador & $1,985,294$ & 16 & 124,080 & 363,846 & 22,740 \\
Santa Ana & 551,259 & 6 & 91,876 & 101,198 & 16,866 \\
San Miguel & 480,276 & 5 & 96,055 & 84,830 & 16,966 \\
La Libertad & 682,1992 & 5 & 136,418 & 132,191 & 26,438 \\
Usulután & 338,332 & 5 & 84,583 & 71,297 & 17,824 \\
Sonsonate & 450,116 & 4 & 112,529 & 91,377 & 22,844 \\
La Unión & 289,021 & 4 & 96,340 & 43,402 & 14,467 \\
La Paz & 292,887 & 3 & 97,629 & 66,196 & 22,165 \\
Chalatenango & 196,583 & 3 & 65,527 & 50,882 & 16,960 \\
Cuscallán & 202,951 & 3 & 67,650 & 44,960 & 14,986 \\
Ahuachapán & 319,780 & 3 & 106,593 & 58,223 & 19,407 \\
Morazán & 173,499 & 3 & 57,833 & 39,817 & 13,272 \\
San Vicente & 161,105 & 3 & 53,701 & 32,804 & 10,934 \\
Cabañas & 152,842 & 3 & 50,947 & 29,246 & 9,748 \\
\hline \multicolumn{1}{c}{ Totales } & $6,276,037$ & 64 & 98,063 & $1,210,269$ & 18,910 \\
\hline
\end{tabular}

- Proyección para el año 2000. "Magnitud o tamaño de la circunscripción (diputados asignados). " Población/ diputados.

Fuentes: elaboración propia sobre datos de la DIGESTYC (1996) y el Tribunal Supremo Electoral (2000a).

12. Las cursivas son del autor.

13. Prácticamente todos los sistemas electorales del mundo provocan efectos desproporcionales por los cuales la proporción de votos que obtienen los partidos no coincide con la proporción de escaños que les son asignados. Un sistema electoral es más proporcional cuanto más se aproxima a uno la relación entre escaños y votos. Cuanto más se aleja de dicho valor esta relación (escaños/votos), más desproporcional es el sistema.

14. El Artículo 12 del Código Electoral asigna los 84 diputados a las 15 circunscripciones territoriales (14 departamentales y 1 nacional), pero no establece el criterio de asignación. Curiosamente, en el caso de los regidores de los concejos municipales, sí queda explícitamente establecida la relación población/regidores. 
Por otra parte, con esta población y con esta distribución de diputados, hay departamentos sobre y subrepresentados. Los diputados de San Salvador, La Libertad, Sonsonate y Ahuachapán representan al doble o más de habitantes que los diputados de Morazán, San Vicente y Cabañas. El primer grupo de departamentos está subrepresentado. Desde el punto de vista de la razón de representación, Morazán, San Vicente y Cabañas deberían tener un diputado menos, es decir dos en lugar de tres.

Podría hacer el análisis no con la población total, sino con la mayor de 18 años, en tanto constituyen la ciudadanía y los potenciales electores. También podría hacer el análisis respecto a los inscritos en el padrón y a los carnetizados. Es muy probable que llegue a similares conclusiones. Por razones de espacio, haré dicho análisis solamente con base en los votos válidos. Al observar el cociente electoral para cada departamento, vuelve a aparecer el fenómeno de la sobre y subrepresentación de unos respecto de otros. Me interesa destacar que este problema no se genera en la fórmula electoral principalmente. Para nada la he considerado hasta este momento $y$, sin embargo, ya tenemos problemas con el reparto de los diputados porque éstos "no valen" lo mismo, ni representan a la misma cantidad de población en todos los departamentos.

$\mathrm{Si}$ atendemos ahora al reparto de escaños entre los partidos, el problema de la sobre o subrepresentación de estos últimos nos damos cuenta que no es algo nuevo lo ocurrido en el 2000 . Una mirada de nuevo al Cuadro 5 permite ver que en todas las elecciones, desde 1982 ha habido partidos sobre o subrepresentados. Y si centramos nuestra atención en 1985, vemos como el PCN obtuvo el 20 por ciento de la representación parlamentaria con el 8.4 por ciento de los votos. ¡Una distancia mayor que la que parece sorprender en el 2000!.(11.6 puntos de diferencia entre escaños y votos en 1985 frente a 7.5 en el 2000). ¿Fue el sistema de residuos la causa de semejante desvia- ción? ¡Definitivamente que no! La explicación está en la política de alianzas practicada por el PCN y ARENA en ese año electoral. Con esto no estoy afirmando que esa sea la causa de la sobrerrepresentación del PCN en el 2000. Pero estoy afirmando que la causa de una sobrerrepresentación de cualquier partido no debe verse sólo en la fórmula electoral y que ésta, aun teniendo alguna influencia, no es la de mayor peso.

\section{Cuadro 17}

Proporcionalidad del sistema electoral salvadoreño 1982-2000

\begin{tabular}{cc}
\hline Año & $\begin{array}{c}\text { Índice de } \\
\text { Rose }\end{array}$ \\
\hline 1982 & 94.2 \\
1985 & 86.9 \\
1988 & 95.9 \\
1991 & 93.5 \\
1994 & 92.8 \\
1997 & 93.6 \\
2000 & 92.4 \\
\hline Promedio & 92.8 \\
\hline
\end{tabular}

Fuente: elaboración propia.

El Cuadro 17 muestra la proporcionalidad que logra nuestro sistema electoral parlamentario. Utilizo para ello el índice de Rose ${ }^{15}$. Lo dicho en el párrafo anterior para la elección de 1985 , se ve reflejado en el valor que asume este índice para ese año (86.9); el de menor proporcionalidad para toda la serie. El valor del índice de Rose para el 2000 es muy cercano al promedio para todo el período; desde este punto de vista, el reparto de escaños en el 2000 no debiera extrañar, por cuanto apenas se desvía 0.4 puntos del índice promedio. ¿Qué motivó, entonces, el "escándalo" sobre el reparto de los escaños en este proceso electoral? Parece ser que el mayor beneficiario de este diseño

15. Se calcula con la fórmula $100-\frac{1}{2} \sum|\mathrm{Vi}-\mathrm{Si}|$, donde $\mathrm{Vi}$ y Si son los porcentajes de votos y escaños del "i-ésimo" partido. 
institucional y no el diseño institucional mismo. ¿Por qué no se hizo escándalo en otros años electorales, incluso en 1994, donde la proporcionalidad apenas difiere 0.4 puntos de la obtenida en el 2000 ?

\subsection{Los efectos reductores}

No todos los partidos que participaron en las elecciones del 12 de marzo obtuvieron representación en la Asamblea Legislativa. De hecho, de los diez partidos inscritos, siete se agenciaron diputados. Este paso de más a menos en el número de partidos que entran al reparto de escaños se conoce como efectos reductores del sistema electoral (r). Sin embargo, para fines comparativos se trabaja con el número efectivo de partidos (electorales y parlamentarios) antes que con los partidos realmente existentes ${ }^{16}$.

Para el 2000, el valor de $r$ es de 5.4. En términos comparativos, este valor es pequeño si se

Cuadro 18

Efectos reductores del sistema electoral salvadoreño, 1982-2000

\begin{tabular}{cr}
\hline Año & r \\
\hline 1982 & 5.9 \\
1985 & 3.7 \\
1988 & 11.1 \\
1991 & 9.1 \\
1994 & 11.4 \\
1997 & $--{ }^{-}$ \\
2000 & 5.4 \\
\hline
\end{tabular}

No se registraron pues Ns > Nv por la coalición PDCPD.

Fuente: elaboración propia. toma en cuenta lo ocurrido en otros años electorales (ver Cuadro 18). Ahora bien, el que unos partidos entren o no al reparto de escaños no depende de una norma explícita en la legislación electoral, tal como sucede en los países donde sí se estipulan barreras legales, por ejemplo, un porcentaje de los votos válidos. En el caso salvadoreño se da, más bien, la existencia de barreras teóricas (ArtigaGonzález, 1996) ad hoc para cada elección. Estas barreras teóricas se ponen en evidencia cuando un partido o coalición no alcanza escaño alguno (es decir, no entra en el reparto), pero no desaparece porque supera el umbral mínimo exigido para evitar que su inscripción sea cancelada por el Tribunal Supremo Electoral. No ha sido este el caso en esta ocasión.
En 1997, el Movimiento de Unidad (MU) alcanzó un escaño, pero no superó el umbral mínimo para garantizar la continuidad de su inscripción en el Tribunal Supremo Electoral. Una situación parecida ocurrió este año (2000) con el CDU, el cual, pese a colocar tres diputados en la Asamblea Legislativa 2000-2003, dejará de existir al no superar el 6 por ciento de los votos válidos que el Artículo 182 del Código Electoral establece para los partidos que forman coaliciones. Al desaparecer los partidos integrantes del CDU, los diputados propuestos por esta coalición se han convertido automáticamente en "diputados independientes". ¡Cosas de la vida!

\section{A manera de conclusión}

Tres han sido los componentes de todo proceso electoral que he analizado en estas páginas. En primer lugar, la distribución territorial de la participación-abstención del electorado salvadoreño en

16. Los efectos reductores se calculan con la fórmula $\mathrm{r}=100(\mathrm{Nv}-\mathrm{Ns}) / \mathrm{Nv}$, donde $\mathrm{Nv}$ y $\mathrm{Ns}$ son el número efectivo de partidos electorales y parlamentarios, respectivamente. 
las recientes elecciones del 12 de marzo. Para darle una perspectiva temporal, he realizado comparaciones con lo ocurrido en 1994 y 1997. La nota sobresaliente no es tanto la tendencia a la baja en la participación y, por tanto, al incremento de la abstención. Lo que llama la atención es que estas tendencias no guardan fuerte relación con las características socioeconómicas (educación e ingreso) de los territorios. Más bien parecen ser procesos políticos específicos los que guardan relación con los niveles de participación registrados en las diferentes zonas geográficas del país. Queda abierta así una línea de investigación para trabajos posteriores.

En segundo lugar, he examinado si es correcto afirmar que en El Salvador existe un bipartidismo. Desde el punto de vista de la competencia electoral y de la asignación de escaños, se trata de una afirmación simplista, que ignora diversos niveles de competencia y, por tanto, la posibilidad de diversos sistemas de partidos en El Salvador. En general, más que bipartidismo, tenemos multipartidismos a varios niveles. El reconocimiento de este "dato" abre las puertas para una reforma electoral que permita la existencia de partidos departamentales, que garantizen la representación de intereses diversos a los del centro nacional. En otras palabras, señala una "puerta institucional" para darle mayor margen de maniobra a los intereses locales (municipales y departamentales) frente a los intereses de la capital (San Salvador) y de la disputa presidencial. También aquí queda abierta otra línea de investigación, que indague sobre las relaciones entre lógicas nacionales y locales en la disputa y el ejercicio del poder político.

Finalmente, las elecciones del 12 de marzo recién pasado abrieron, una vez más, la caja de Pandora. Así, la principal sorpresa para muchos fue el número de diputados logrados por el PCN, gracias al sistema electoral. En este trabajo he insistido en que no debería ser eso lo que llame la atención pues, en perspectiva temporal, no ha sido realmente una sorpresa. Nuestro sistema electoral está diseñado para favorecer un sistema de tres partidos a nivel nacional. Cualquier partido que sea la tercera fuerza electoral tendrá siempre un mayor bono de representación en la Asamblea Legislativa. No tanto por la fórmula utilizada, sino por la distribución territorial de los escaños. No solamente estamos ante problemas de desproporcionalidad votos-escaños, sino también ante la violación del principio democrático de igualdad en el valor del voto (una persona $=$ un voto). Esto queda patente en el análisis territorial de la población y la asignación de diputados que a cada territorio le concede el Código Electoral. ¡Allí comienza ya el problema y no en el último eslabón!

\section{Bibliografia}

Artiga-González, Álvaro (1999a). "Competitividad y participación electoral en El Salvador, 1982-1999". En Realidad, 69, San Salvador: Universidad Centroamericana "José Simeón Cañas", pp. 275-290.

Artiga-González, Álvaro (1999b). La política y los sistemas de partidos en Centroamérica. (Tesis de Doctorado en Ciencia Política.) Universidad de Salamanca, España.

Artiga-González, Álvaro (1998). “Fluidez y Volatilidad en la institucionalización de los sistemas de partidos (Notas metodológicas para su medición)". En América latina hoy, 19, Salamanca: Instituto de Estudios de Iberoamérica y Portugal y el Seminario de Estudios Políticos sobre Latinoamérica, pp. 125-129.

Artiga-Gonzálcz, Álvaro (1997a). "Aproximación al comportamiento electoral de los centroamericanos". En Realidad, 59, San Salvador: Universidad Centroamericana José Simeón Cañas, pp. 459-475.

Artiga-González, Álvaro (1997b). "Efectos de los sistemas electorales en El Salvador". En Realidad, 58, San Salvador: Universidad Centroamericana José Simeón Cañas, pp. 333-346.

Artiga-González, Alvaro (1996a). El Salvador: el ascenso electoral del partido ARENA y las elecciones del siglo. (Tesis de Maestría en Ciencias Sociales.) México: FLACSO.

Artiga-González, Álvaro (1996b). "Sistemas electorales en El Salvador". En Realidad, 54, San Salvador: Universidad Centroamericana José Simeón Cañas, pp. 765-788.

Artiga-González, Álvaro (1993). "Buscando la clave de las elecciones del 94". En Realidad, 39, San Salvador: Universidad Centroamericana José Simeón Cañas, pp. 443-458.

CACH-PROCHALATE (1999). Bases para el desarrollo sostenible de Chalatenango. Plan departamental de manejo ambiental (PADEMA). Chalatenango: CACH-PROCHALATE.

CIDAI (2000). "Las elecciones del 12 de marzo: resultados preliminares". En Proceso, 894. San Salvador: Centro de Información, Documentación y Apoyo a la Investigación. 
Delgado Sotillos, Irene (1997). El comportamiento electoral municipal español, 1979-1995. Madrid: Centro de Investigaciones Sociológicas.

DIGESTYC (1996). Proyección de la población de El Salvador. San Salvador: Dirección General de Estadística y Censos, Ministerio de Economía.

El Diario de Hoy (12/3/2000). Elecciones 2000: Urnas abiertas para más de 3 millones de electores, San Salvador.

Enríquez, Alberto et al. (1997). Estudio de caso: Comité Ambiental de Chalatenango-CACH. San Salvador: FUNDE.

Justel, Manuel (1995). La abstención electoral en España, 1977-1993. Madrid: Centro de Investigaciones Sociologicas.

La Prensa Gráfica (10/3/2000). Elecciones 2000, San Salvador, pp. 32-49.
Laakso, Markku y Taagepera, Rein (1979). "Efective Number of Parties. A Measure with Application to West Europe". En Comparative Political Studies, 1, Vol. 12, pp. 3-27.

PNUD (1997). Informe sobre indices de Desarrollo $\mathrm{Hu}$ mano en El Salvador. San Salvador: Dirección General de Estadística y Censos.

Sartori, Giovanni (1992). Partidos y sistemas de partidos. Madrid: Alianza.

Tribunal Supremo Electoral (2000a). Resultados de los escrutinios finales de la Asamblea Legislativa, por departamento.

Tribunal Supremo Electoral (2000b). Resultados de los escrutinios finales de la Asamblea Legislativa, por municipio.

Vaquerano, Ricardo (2000). "PCN: menos votos pero más diputados". En La Prensa Gráfica, 15 de marzo de 2000, San Salvador, pp. 12-13. 